\title{
Hybrid Precoding for Millimeter Wave RoF Systems
}

This paper was downloaded from TechRxiv (https://www.techrxiv.org).

\section{LICENSE}

CC BY 4.0

SUBMISSION DATE / POSTED DATE

09-04-2020 / 10-04-2020

\section{CITATION}

Alghorani, Yahia; Ikki, salama (2020): Hybrid Precoding for Millimeter Wave RoF Systems. TechRxiv. Preprint. https://doi.org/10.36227/techrxiv.12107043.v1

DOI 


\section{Hybrid Precoding for Millimeter Wave RoF Systems}

\author{
Yahia Alghorani \\ Department of Electrical Engineering \\ Lakehead University \\ Ontario, Canada \\ yahia.alghorani@ieee.org
}

\author{
Salama Ikki \\ Department of Electrical Engineering \\ Lakehead University \\ Ontario, Canada \\ sikki@lakeheadu.ca
}

\begin{abstract}
Large propagation path loss and limited scattering of millimeter wave (mm-wave) channels create new challenges for physical layer signal processing. Hence, in this paper, we propose a photonic hybrid precoding model for $\mathbf{m m}$-wave radio-over-fiber systems that overcomes hardware constraints on radio frequency precoding. With the help of analog photonic beamformers, the hybrid precoding strategy combats the large losses of mm-wave and mitigates inter-user interference, which in turn reduces the number of RF chains required to perform digital beamforming. The simulation results show that the proposed model can offer significant coding gains over conventional RF hybrid precoding systems. Unlike RF hybrid precoding systems, the photonic hybrid precoding systems can provide higher data rates and lower error rates in multi-user transmission scenarios.
\end{abstract}

Index Terms-Hybrid precoding, mm-wave RoF systems.

\section{INTRODUCTION}

Hybrid analog-digital precoding transceivers have recently attracted considerable attention for millimeter wave (mmwave) multiple-input multiple output (MIMO) systems [1],[2]. This type of transceiver requires only a small number of radio frequency (RF) chains (e.g., signal mixers, highly linear power amplifiers, filters, and high-resolution digital-to-analog (DAC) converters) to provide high precoding (beamforming) gains and combat large path loss of mm-wave signals [3]. Because of the high-power consumption of mm-wave circuit components and nonlinear electric phase shifters, as well as the feedback overhead of complete knowledge of channel state at the transmitter, the performance of conventional RF hybrid precoding systems becomes limited when multi-user interference is considered in small-cell environments. Thus, radio-over-fiber (RoF) technology has become a promising solution to overcome the limitations of RF devices and to increase network capacity [4]. Currently, the next generation (5G) mobile networks operating in millimeter wave frequency bands of $30 \mathrm{GHz}$ to $110 \mathrm{GHz}$ are proposed to deliver higher capacity and bandwidth than current $4 \mathrm{G}$ networks. In $5 \mathrm{G}$ deployments, the network is also expected to have higher coverage, higher bandwidth and lower latency, lower battery consumption, and higher density of mobile users, for better implementation of the internet of things (IoT) technology [5]. The use of RoF communication systems (such as elastic radiooptical network (ERON)) for the backhaul and fronthaul access networks will become one of the most efficient solutions to enhance spectral efficiency of mm-wave communication systems and mitigate inter-user interference. Considering channel coding techniques for long-haul fiber optics communications, such as low-density parity-check (LDPC) codes and turbo

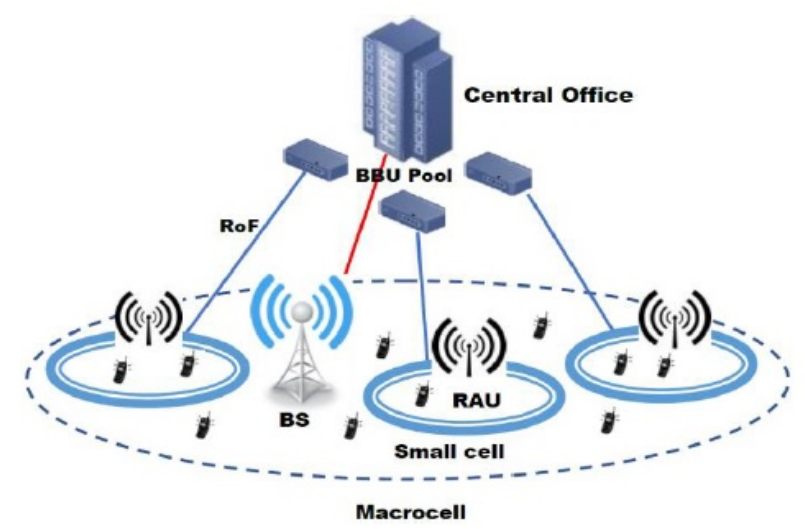

Fig. 1: Illustration of ERON architecture

codes, the RoF systems become more efficient to increase the spectral efficiency while transmitting a message over a noisy transmission channel [6],[7]. The ERON topology (see Fig.1) can deliver elastic networking through central wireless communication systems by deploying EON in a wide-metro area network (WAN-MAN) and by introducing microwaveoptical signal processing into the local area network (LAN). Generally, the purpose of the ERON structure is to localize digital base-band units (BBUs) in the central office to reduce the complexity of conventional cell sites and to deploy a small cell system on a large scale [8]. Since the BBU is shifted to the central office, RF signals are generated at the BBU and transmitted to remote access units (RAUs) through optical fibers, so that low-loss wideband signals can be transported over long distances with an increase in system capacity. The key advantages of ERON are 1) achieving low-cost, low-noise, low-power, and high-fidelity RF-optical signal processing via silicon photonic integrated circuits (Si-PICs) [9], while replacing the power-hungry $\mathrm{mm}$-wave electronic circuitry and nonlinear electric phase shifters. 2) Unlike the baseband-overfiber $(\mathrm{BoF})$ approach that supports only one frequency band at a time, the RoF approach provides multiple bands multiple carrier signals so that multiple operators can coexist in a shared infrastructure without interference [4]. However, the relatively large propagation loss and limited scattering of mm-wave create new challenges for physical layer signal processing. To address these challenges, we propose a hybrid architecture that uses RF-optical processing units (RF-OPUs) to provide steerable beams and compensate the large propagation loss of mm-wave [10]. RF-optical processing components have 

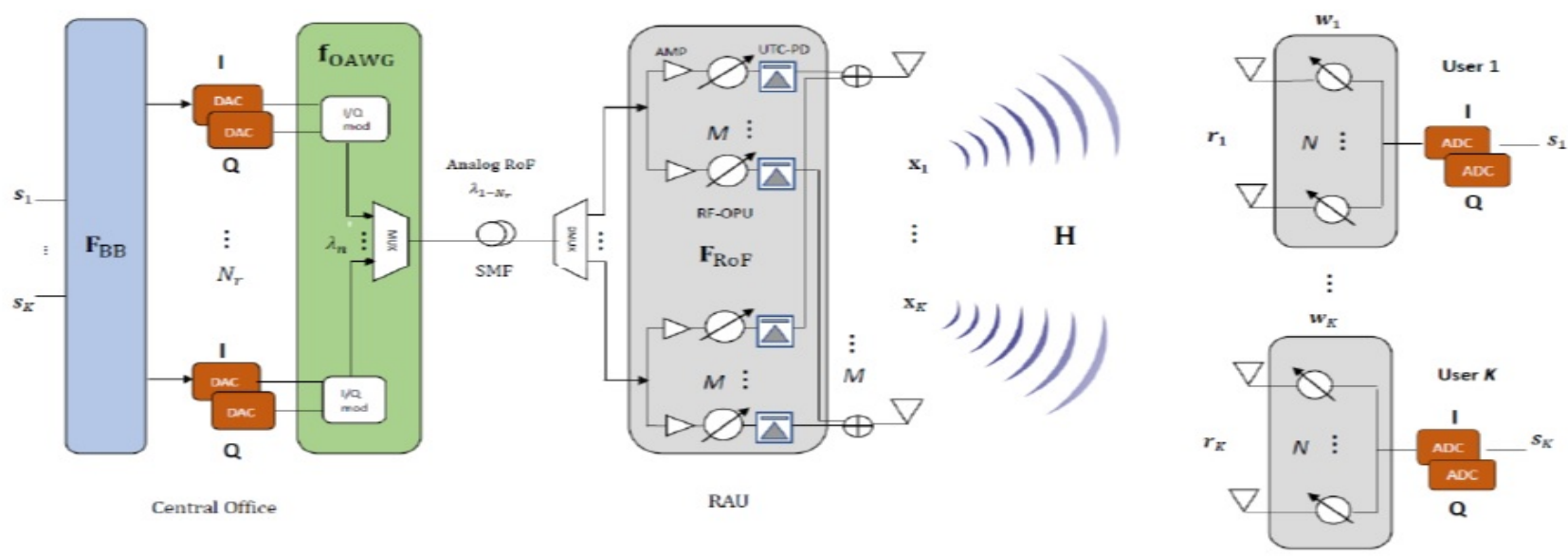

Fig. 2: Photonic hybrid precoding structure for mm-wave multi-user MIMO systems (where $\mathbf{H} \in \mathcal{C}^{N \times M}$, is the channel impulse response model for the $K$ users)

a wide range of applications due to high bandwidth, low latency and potential low cost compared to all electronic processing [11]. Rather than using electrical components to form unit cells in electrical lattice filters, optical taps, delay lines, and resonators with tuning elements can form unit cells in silicon optical lattice filters for filtering, equalization, and amplitude/phase tuning in the optical domain with RF frequency resolutions (10-100 MHz) [11], [12]. In mm-wave communications, the silicon photonic lattice filter array can 1) supply steerable beams to compensate for large path-loss and interference removal by boosting the signal across multiple carriers provided by RoF systems; 2) achieve high spectral efficiency through MIMO systems. With directional beams in the RF optical layer, the number of antenna elements at the RAU that are needed to create narrow beams is reduced, and a low-rank channel estimate is performed. Since the antenna array size is very small compared to the distance between the RAU and the mobile terminal, we can set the same analog photonic beamformer for different streams of the same user in the same frequency band; the RoF signal is then divided into multiple sub-band signals and transmitted on an appropriate beam. In this way, we can simplify the design of weighting coefficients of beam steering vectors, which are required to calculate the beam steering angle. However, most existing works have focused on conventional RF hybrid precoding systems (see e.g., [1-3]). To the best of our knowledge, the photonic hybrid precoding of RoF systems has not been studied before; therefore, in this study, we propose a new mm-wave RoF system structure (hybrid precoding-based) that can bring connection ubiquity, mobility, and interference suppression in multi-user scenarios. Specifically, the paper's contributions are summarized as follows:

- Improving coding gains of conventional RF hybrid precoding systems up to $r=M N_{r}(K-1) / L K^{2}$ and developing a low-complexity system architecture for multi-user MIMO transceivers, which can use a small number of $\mathrm{RF}$ chains (DACs/ADCs) to transmit multi-data streams at low hardware costs and power consumption.

- Unlike conventional RF hybrid precoding systems which are limited to narrow-band signals, the photonic hybrid precoding systems can provide a higher data rate and lower bit error rate when the number of RF chains is equivalent to the number of users.

- In order to combat interference in ultra-dense small cells scenarios, our proposed scheme can maximize the signal energy (i.e., low-resolution ADCs to recover the $\mathrm{mm}$-wave signals) associated with each bit of user data by performing beam steering with the analog photonic beamformers (where steerable beams can be obtained at $\mathrm{mm}$-wave), so that the large training overhead required for accurate channel estimation is reduced, and inter-cell interference between users is avoided.

\section{Notation:}

Boldface symbols denote matrices $\mathbf{A}$ and vectors $\boldsymbol{a}$. The superscripts $(.)^{T}$ and $(.)^{*}$ stand for transpose and conjugate, transpose operatars, respectively. (. $)^{-1}$ denotes the matrix inverse. The determinant and trace operators are denoted by $\operatorname{det}($.$) and \operatorname{Tr}($.$) . I denotes the identity matrix and \operatorname{diag}($. denotes a diagonal matrix, $|$.$| is the magnitude of a scalar,$ and $\|\cdot\|_{F}$ stands for the Frobenius norm. The Moore-Penrose pseudoinverse operator is denoted by $(\cdot)^{+}$.

\section{System And Channel Model}

We consider a mm-wave multi-user MIMO system equipped with $M$ transmit and $N$ receive antennas per user. The system is operating in the ERON and consisting of a digital baseband precoder, $\mathbf{F}_{\mathrm{BB}} \in \mathcal{C}^{K \times N_{s}}$, located at the central office, and an analog photonic precoder (beamformer), $\mathbf{F}_{\mathrm{RoF}} \in C^{M \times N_{r}}$, at the transmitter (RAU), while the receiver at user $k$ uses only an analog electronic combiner, $\boldsymbol{w}_{k} \in \mathcal{C}^{N \times 1}$ for $k=$ $1,2 \ldots, K$, as shown in Fig. 2. At the output of the baseband precoder, the data streams for the $K$ users (i.e, $s \in \mathcal{C}^{K \times 1}$ where $\boldsymbol{s}_{k} \in \mathcal{C}^{N_{s} \times 1}$ is the vector of information-bearing symbols for transmission to user $k$ ) are carried on $N_{r}$ optical carriers and precoded into a set of $N_{r}$ RF chains (DACs) which is subject to the constraint [1] $K \leq N_{r} \leq M$. Each DAC is connected to a dynamic optical arbitrary waveform generator (OAWG), which is, in turn, used to generate optical 

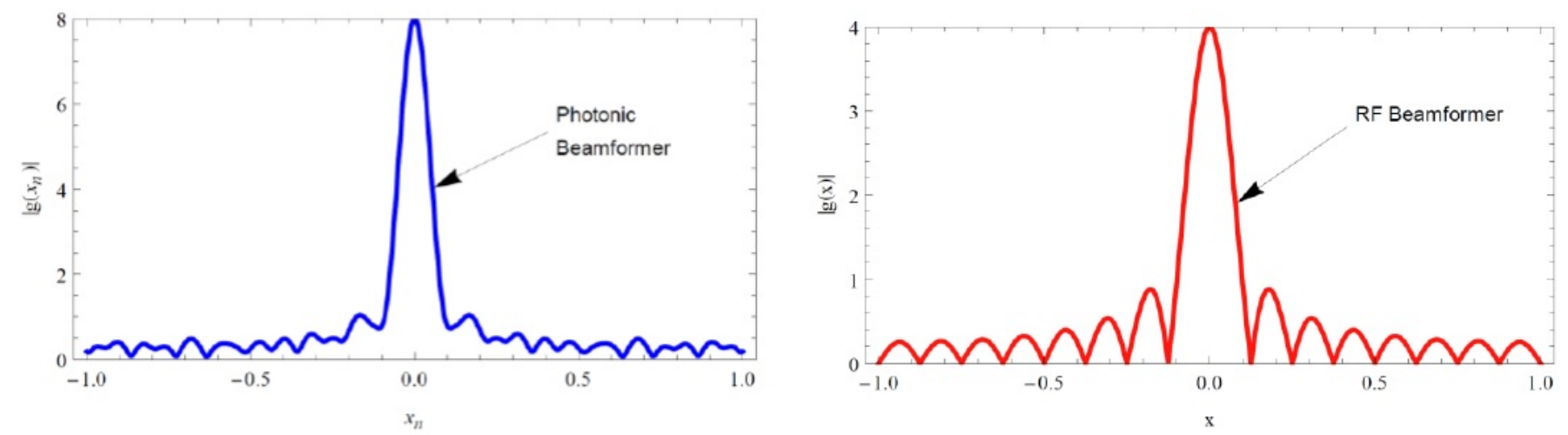

Fig. 3: Comparison between the array gain of RF and photonic beamformers. A fine beam pattern for each user, generated by 16 antenna elements with an element spacing of $\lambda_{\max / 2}$, for a carrier frequency $28 \mathrm{GHz}$. The analog photonic beamformer assigns a set of 4 wavelengths $\left(\lambda_{1}=10.70 \mathrm{~mm}, \lambda_{2}=7.1 \mathrm{~mm}, \lambda_{3}=4.99 \mathrm{~mm}, \lambda_{4}=4.10 \mathrm{~mm}\right)$ used by each antenna element in the array, while the RF beamformer employs a single-wavelength $(\lambda=10.70 \mathrm{~mm})$. The maximum array gain ratio is $\left|g\left(x_{n}\right)\right| /|g(x)|=\sqrt{N_{r}}$

carrier wavelengths $\lambda_{n}$ (where $n=1,2, \ldots, N_{r}$ ) through an in-phase and quadrature (IQ) optical intensity modulators array, $\mathbf{f}_{\mathrm{OAWG}} \in \mathcal{C}^{N_{r} \times 1}$ [11]. Correspondingly, the IQ modulators carry the data streams over RF signals which then transported by a single mode optical fiber (SMF). At the transmitter, the analog photonic beamformer $\mathbf{F}_{\mathrm{RoF}}$ employs Si-PICs consisting of an optical power splitter, an optical amplifier array, RF-OPUs for phase/amplitude tuning, and unit-traveling carrier photo-detectors (UTC-PDs) to produce the mm-wave signals [13]. We use the UTC-PDs to generate the mm-wave signals via the photonic signals that are optically amplified without involving mm-wave circuits (e.g., mm-wave upconverters). While the generated OAWG waveform is at optical carrier frequencies, direct square-law detection by UTC-PDs can provide coherent RF mixing functions in the optical domain without involving mm-wave electronics [14], [15]. Such UTC-PDs are supplied by NTT Electronics and sold as mm-wave photomixers with or without integrated antennas, covering up to $170 \mathrm{GHz}$. Given the system model, our main target focuses on adaptive beam steering and interference cancellation through the analog photonic beamformers.

For simplicity, we adopt unit-gain omni-directional antennas at both RAU and users, and we use the widely accepted geometric mm-wave channel model based on the extended Saleh Valenzuela model [16], where we assume that there is a narrowband-block fading propagation channel model with a constant mm-wave channel matrix, $\mathbf{H}_{k} \in \mathcal{C}^{N \times M}$, between the RAU and user $k$. Unlike conventional microwave systems; in order to achieve a high spatial multiplexing gain with low-complexity signal processing, the RAU can rely on the characteristics of a multi-carrier optical transmission when accurate channel knowledge is limited due to estimation errors and quantization. However, accurate and timely acquisition of channel state information (CSI) is challenging, especially in inter-user interference and high mobility scenarios [17]. Thus, our proposed scheme can offer a significant gain in network throughput with a low-cost hardware implementation.

In multi-user MIMO systems where the interference between the multiplexed data streams takes place, the received signal vector, $\boldsymbol{r}_{k} \in \mathcal{C}^{N \times 1}$, at user $k$, is given as $\boldsymbol{r}_{k}=$ $\sqrt{\rho} \sum_{m=1}^{K} \mathbf{H}_{k} \mathbf{x}_{m}+\mathbf{n}_{k}, k \in\{1,2, \ldots, K\}$, where $\mathbf{n}_{k} \in \mathcal{C}^{N \times 1}$ is the additive white Gaussian noise (AWGN) vector with entries having zero mean and variance $N_{o}$. The received signal is processed by the analog electronic combiner $\boldsymbol{w}_{k}$ to obtain $\mathrm{y}_{k}=\boldsymbol{w}_{k}^{H} \boldsymbol{r}_{k}$, which can be rewritten as

$$
\mathrm{y}_{k}=\sqrt{\rho} \boldsymbol{w}_{k}^{H} \mathbf{H}_{k} \mathbf{x}_{k}+\sqrt{\rho} \sum_{m=k}^{K} \boldsymbol{w}_{k}^{H} \mathbf{H}_{k} \mathbf{x}_{m}+\boldsymbol{w}_{k}^{H} \mathbf{n}_{k}
$$

where $\rho=P_{s} / K$ is the transmit signal power per user, $\mathbf{x}_{k}=\mathbf{F}_{\mathrm{RoF}} \mathbf{f}_{\mathrm{OAWG}} \mathbf{F}_{\mathrm{BB}}^{(k)} s_{k}$ is the signal vector $\left(\mathbf{x}_{k} \in \mathcal{C}^{M \times 1}\right)$ transmitted by the RAU to user $k$ (where the sum of signal vectors that appears in (1), is the additional interference received from other users), and $\mathbf{F}_{\mathrm{BB}}^{(k)}$ is the $k$-th row of the $\mathbf{F}_{\mathrm{BB}}$ matrix. The normalized total transmit power constraint can be given by $\left\|\mathbf{F}_{\mathrm{RoF}} \mathbf{f}_{\mathrm{OAWG}} \mathbf{F}_{\mathrm{BB}}\right\|_{F}^{2}=N_{r} K$. Therefore, the received signal-to-interference-plus-noise ratio (SINR) at user $k$ becomes

$\operatorname{SINR}_{k}=\frac{\rho\left|\boldsymbol{w}_{k}^{H} \mathbf{H}_{k} \mathbf{F}_{\mathrm{RoF}} \mathbf{f}_{\mathrm{OAWG}} \mathbf{F}_{\mathrm{BB}}^{(k)} \boldsymbol{s}_{k}\right|^{2}}{\rho \sum_{m=k}^{K}\left|\boldsymbol{w}_{k}^{H} \mathbf{H}_{k} \mathbf{F}_{\mathrm{RoF}} \mathbf{f}_{\mathrm{OAWG}} \mathbf{F}_{\mathrm{BB}}^{(m)} \boldsymbol{s}_{m}\right|^{2}+\left\|\boldsymbol{w}_{k}\right\|^{2} N_{o}}$

The corresponding link spectral efficiency is $\log _{2}\left(1+\mathrm{SINR}_{k}\right)$ bits/sed Hz, where the digital precoder employs Zero-forcing (ZF) precoding, which is selected as [18]

$$
\mathbf{F}_{\mathrm{BB}}^{(k)}=\mathbf{H}_{\mathrm{p}(k)}^{\dagger}=\mathbf{H}_{\mathrm{p}(k)}^{H}\left(\mathbf{H}_{\mathrm{p}(k)} \mathbf{H}_{\mathrm{p}(k)}^{H}\right)^{-1}
$$

where $\mathbf{H}_{\mathbf{p}(k)}=s_{k} \boldsymbol{w}_{k}^{H} \mathbf{H}_{k} \mathbf{F}_{\mathrm{RoF}} \mathbf{f}_{\mathrm{OAWG}} \cdot$ The ZF precoder aims at nulling inter-user interference but in return neglects the effect of noise, which means that it works poorks poorly under noise-limited scenarios. In contrast, minimum mean-square error (MMSE) precoding can provide a tradeoff between noise amplification and interference suppression, at the expense of increasing system complexity. This precoder can be expressed as

$$
\mathbf{F}_{\mathrm{BB}}^{(k)}=\mathbf{H}_{\mathrm{p}(k)}^{H}\left(\mathbf{H}_{\mathrm{p}(k)} \mathbf{H}_{\mathrm{p}(k)}^{H}+\frac{\left\|\boldsymbol{w}_{k}\right\|^{2} \mathbf{I}_{K}}{\mathrm{snr}}\right)^{-1}
$$

where the signal-to-noise ratio (SNR) is defined as snr = $P_{s} / N_{o}$. In general, both the linear $\mathrm{ZF}$ and MMSE precoders 
may achieve a full multiplexing gain if the complete CSI is fed back to the transmitter with good accuracy, thus, the photonic beamformers-based RoF systems can compensate the signal power loss caused by imperfect CSI at the transmitter, through spreading multiple data streams over multiple carriers. Since the mm-wave channels have large propagation path loss and limiting scattering, we use a geometric channel model between the RAU and the user, described by [19]

$$
\mathbf{H}_{k}=\sqrt{\frac{M N}{L_{k}}} \sum_{l=1}^{L_{k}} \alpha_{k, l} \boldsymbol{a}_{r}\left(\theta_{k, l}\right) \boldsymbol{a}_{t}^{H}\left(\emptyset_{k, l}\right)
$$

where $L_{k}$ is the number of scatters at user $k, \alpha_{k, l}$ is the complex gain of path $l, \theta_{k . l} \in[0,2 \pi]$ and $\emptyset_{k . l} \in[0,2 \pi]$ are the angle of arrival (AoA) and the angle of departure (AoD), respectively. It is often useful to represent the channel in the frequency domain rather than the time domain, where the channel varies slowly during the signal duration and Doppler shifts for all paths are small [1, Eq. (6)]. In case the channel bandwidth is small, we approach (4). With uniform linear arrays (UILA), the $k$-th array response vector at the RAU is expressed as

$$
\begin{aligned}
\boldsymbol{a}_{t}\left(\emptyset_{k, l}\right) & =\frac{1}{\sqrt{M}}\left[1, e^{j\left(2 \pi / \lambda_{n}\right) d \sin \left(\emptyset_{k}, l\right)}, \ldots\right. \\
& \left.\times \ldots, e^{j(M-1)\left(2 \pi / \lambda_{n}\right) d \sin \left(\emptyset_{k, l}\right)}\right]^{T}
\end{aligned}
$$

where $d$ is the distance between antenna elements and $\lambda_{n}$ is the wavelength of the carrier $n$. A similar expression can be written for the array response vector at user $k, \boldsymbol{a}_{r}\left(\theta_{k, l}\right)$. Since the wavelengths of mm-wave signals are very small, the antenna elements of ULA can be designed at $d=\lambda_{\max / 2}$, for both the transmitter and receiver. By using the photonic phase shifters (which are fixed into the RF-OPUs) at the array steering vector (ULA), we can model the analog photonic beamformer $\mathbf{F}_{\text {RoF }}$ per optical carrier $\lambda_{n}$, as

$\mathbf{F}_{\mathrm{RoF}}^{(n)}=\left[1, e^{j\left(2 \pi / \lambda_{n}\right) d \sin \left(\emptyset_{k, o}\right)}, \ldots, e^{j(M-1)\left(2 \pi / \lambda_{n}\right) d \sin \left(\emptyset_{k, o}\right)}\right]^{T}$

where $\phi_{k, 0}$ is the $k$-th beam focus angle (steering angle), in which the photonic beamformer can achieve the highest array gain.

\section{PROBlem Formulation}

Because of the high-frequency band of mm-waves, conventional RF beamformers [1] (electric phase shifters-based) cause phase shifter loss, high noise power, and nonlinearity, which results in beam squint (steering error) across the frequency. Additionally, the use of high mm-wave bandwidth leads to high noise power and low received SNR, which makes it difficult to implement functions like channel estimation and beam training. Since mm-wave signals occupy a very small portion of the optical bandwidth, linear RF phase shifts are achievable by using a linear photonic filter [20]. Research studies have demonstrated that RF-photonic technologies can perform a) low relative-intensity-noise lasers, and b) highly linear optical modulators and photo-detectors even at high power levels [21], [22]. All these aspects are significant when realizing high-performance RoF links with high SNR values. Therefore, our goal of using the analog photonic beamformer is to maximize the SNR by eliminating noise and inter-user interference. Given the array steering vector in (6), the $k$-th array gain of the analog photonic beamformer, $g_{k}=\frac{1}{\sqrt{N_{r}}} \Sigma_{n} a_{t}^{H}\left(\emptyset_{k, l}\right) \mathbf{F}_{\mathrm{RoF}}^{(n)}$ expressed as

$$
g\left(x_{k, n}\right)=\frac{1}{\sqrt{M N_{r}}} \sum_{n=1}^{N_{r}} \sum_{m=1}^{M} e^{j(m-1)\left(2 \pi / \lambda_{n}\right) d x_{k, n}}
$$

where $x_{k, n}=\xi_{n} \sin \left(\phi_{k, n}\right)-\sin \left(\phi_{k, 0}\right)^{1}$ models the beam squint effect for line-of-sight (LOS) scenarios (i.e., $L=1$ ), and $\phi_{k, n}$ is the $k$-th AoD for the carrier $f_{n}$. In contrast, the $k$-th array gain of the narrowband (i.e., single-carrier) RF beamformer, is expressed as

$$
g\left(x_{k}\right)=\frac{1}{\sqrt{M}} \sum_{m=1}^{M} e^{j(m-1)(2 \pi / \lambda) d x_{k}}
$$

From (7) and (8), the phase shift causes different frequency components to be steered to different angles. Meaning that the beam steering errors increase if the $\mathrm{AoD} / \mathrm{AoA}$ gets away from the desired beam steering angle $\phi_{k, 0}$. To get further insight into the performance of the photonic beamformer, we need to find an asymptotic expression for the $k$-th array gain. Assuming the antenna elements of the analog photonic beamformer have the same amplitude [23], where a fine beam pattern is achieved through the optical carriers $\lambda_{n}$, then using the inequality $e^{a x} \geq$ $(1+x)^{a} \geq 1+a x$ when $x \rightarrow 0$, the photonic array gain can be bounded as $g\left(x_{k}\right) \geq \sqrt{N_{r} / M}\left(1+j(M-1) 2 \pi \lambda^{-1} d x_{k}\right)$, which concludes that the beam squint errors generated by the coefficient $\xi_{n}$, can effectively be minimized when the number of wavelengths $\lambda_{n}$ increases. This would provide a narrow directional beam (i.e., a steerable beam) with higher gain to compensate for attenuation and beam squint in the mm-wave band and reduce the interference effects from other closely users. Fig. 3 illustrates an example fine beam pattern for both the photonic and RF beamformers, where we assume that the phase-shifter array is steered to the desired angle for the carrier frequency (i.e., $\xi_{n}=1$ ). The results show that the analog photonic beamformer can provide a narrow directional beam (i.e., a steerable beam with better tuning for the $\boldsymbol{a}_{t}$ and $\boldsymbol{a}_{r}$ weighting vectors) with a higher gain of signal power, which indicates that the photonic beamformer $\mathbf{F}_{\mathrm{RoF}}$ can combat Doppler shift effects and tackle the low SNR and inter-user interference at mm-waves. In other words, the results show that the analog photonic beamformer $\mathbf{F}_{\mathrm{RoF}}$ can provide a narrow directional beam (i.e., a beam is steerable by sending the users' data streams over multi-carrier frequencies) with a higher gain of signal power, which indicates that the photonic

\footnotetext{
${ }^{1} \xi_{n} \in\left\lceil 1-b_{n} / 2,1+b_{n} / 2\right\rceil$ stands for the ratio of a subcarrier to the carrier frequency $f_{n}$ (i.e., $f=\xi_{n} f_{n}$, where $f \in\left[f_{n}-\frac{B W_{n}}{2}, f_{n}+\frac{B W_{n}}{2}\right], f_{n}=$ $c / \lambda_{n}$, and $c$ is the speed of light), $b_{n}=B W_{n} / f_{n}$ is the fractional bandwidth (where $B W_{n}$ is the baseband bandwidth of the signal).
} 

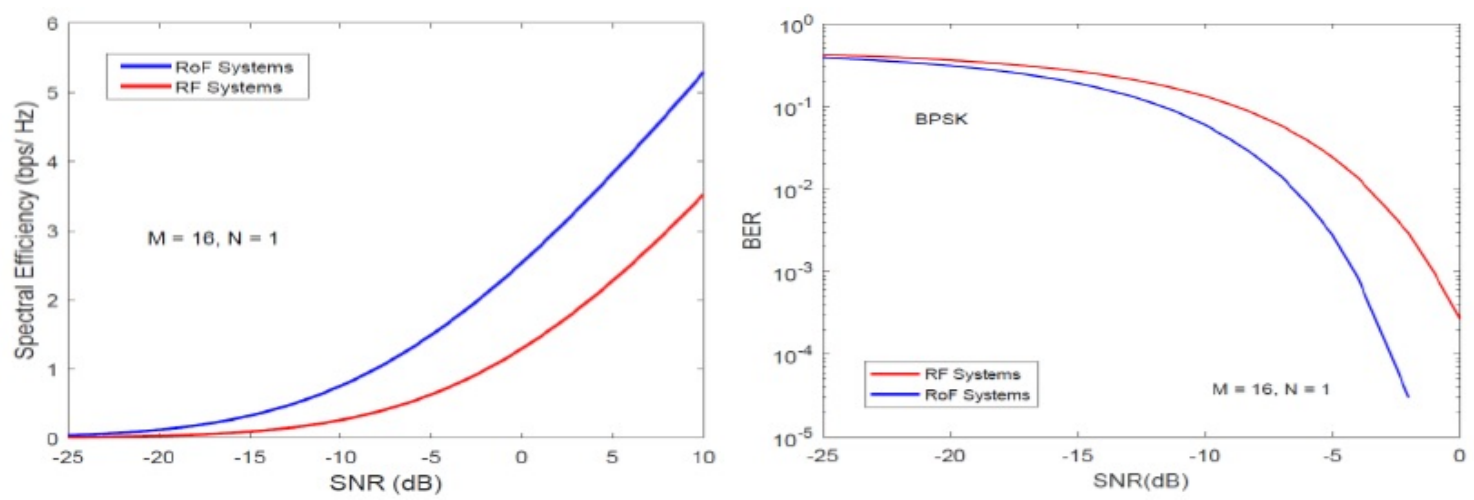

Fig. 4: Spectral efficiency and error probability comparison of RoF systems and conventional RF systems [1] (hybrid precoding-based). Monto-Carlo simulation is implemented at $10^{5}$ channel realizations. In this multi-user transmission scenario, the number of RF chains and optical carriers, is equal to the number of users, e.g., $K=N_{r}=3, \lambda_{1-N_{r}}=d / 2, L=1$ (LOS paths).

beamformer can maximize the received SNR (2) at each user by eliminating noise and inter-user interference.

\section{Photonic Hybrid Precoding for Multi-user MIMO SYSTEMS}

To examine the impact of analog photonic beamformers on the hybrid precoding systems and to determine the minimum number of RF chains required to obtain the minimum spectral efficiency among all users, we investigate the performance of RoF systems when the number of RF chains is equivalent to the number of users (i.e., $N_{r}=K$ ). To simplify the analysis, we focus on the case that each user is equipped with a single antenna (i.e., $N=1$ ). Hence, the received signal vector, $\boldsymbol{r} \in$ $\mathcal{C}^{K \times 1}$, is given by

$$
r=\sqrt{\rho} \mathbf{H} \mathbf{x}+\mathbf{n}
$$

where $\mathbf{x}=\mathbf{F}_{\mathrm{RoF}} \mathbf{f}_{\mathrm{OAWG}} \mathbf{F}_{\mathrm{BB}} \mathbf{S}$ is the transmit signal vector for the $K$ users (where the information-bearing signal vector of user $k\left(s_{k}\right)$ is normalized to unity (i.e., $N_{s}=1$ ), that is, $\mathrm{s} \in$ $\mathcal{C}^{K \times 1}, \mathbf{F}_{\mathrm{BB}} \in \mathcal{C}^{1 \times K}$, and $\left.\mathbf{x} \in \mathcal{C}^{M \times 1}\right), \mathbf{n} \in \mathcal{C}^{K \times 1}$ represents the noise and interference ball vector, and $\mathbf{H} \in \mathcal{C}^{K \times M}$ is the channel response for the $K$ users, which is subject to the constraint of $\|\mathbf{H}\|^{2}=\operatorname{Tr}\left(\mathbf{H} \mathbf{H}^{H}\right)$

$$
\operatorname{Tr}\left(\mathbf{H H}^{H}\right)=\sum_{k} \sum_{l} \frac{M}{L K}\left|\alpha_{k, l}\right|^{2}
$$

\section{A. Achievable Spectral Efficiency}

In our analysis, we focus on the downlink transmission. Plus, we assume that the channel $\mathbf{H}$ is known to the transmitter. The transmit symbols follow a Gaussian distribution with Rayleigh fading, in which achievable spectral efficiency is expressed as

$$
R=\log \operatorname{det}\left(\mathbf{I}_{\mathbf{K}}+\frac{\rho}{N_{o}} \mathbf{H F}_{\mathrm{RoF}} \mathbf{f}_{\mathrm{OAWG}} \mathbf{F}_{\mathrm{BB}} \mathbf{F}_{\mathrm{BB}}^{H} \mathbf{f}_{\mathrm{OAWG}}^{H} \mathbf{F}_{\mathrm{RoF}}^{H} \mathbf{H}^{H}\right.
$$

To maximize the spectral efficiency in (10), we assume that the columns of the digital precoding matrix $\left(\mathbf{F}_{\mathrm{BB}}\right)$ are mutually orthogonal, where the interference between the data streams is canceled, namely

$$
\mathbf{F}_{\mathrm{BB}}^{H} \mathbf{F}_{\mathrm{BB}}=\sigma \mathbf{I}_{K}
$$

Proceeding with the design of the analog photonic precoder, $\mathbf{F}_{\mathrm{RoF}} \mathbf{f}_{\mathrm{OAWG}}$, and the digital precoder, $\mathbf{F}_{\mathrm{BB}}$, the corresponding optimization problem is given by

$$
\begin{array}{r}
\min _{\mathbf{F}_{\mathrm{ROF}}, \mathbf{F}_{\mathrm{BB}}, \sigma}\left\|\mathbf{F}_{\mathrm{opt}}-\mathbf{F}_{\mathrm{RoF}} \mathbf{f}_{\mathrm{OAWG}} \mathbf{F}_{\mathrm{BB}}\right\|_{F}^{2} \\
\text { subject to }\left\{\begin{array}{l}
\mathbf{F}_{\mathrm{RoF}} \in \mathcal{F}_{\mathrm{RoF}} \\
\mathbf{F}_{\mathrm{BB}}^{\mathrm{H}} \mathbf{F}_{\mathrm{BB}}=\sigma \mathbf{I}_{K}
\end{array}\right.
\end{array}
$$

where $\mathbf{F}_{\text {opt }} \in \mathcal{C}^{M \times K}$, stands for the optimal digital precoding (i.e., $\left.N_{r}=M\right)$, and $\mathcal{F}_{\text {RoF }}$ can be defined as a set of weighting coefficients $\left(\emptyset_{k, l}\right)$ for the $K$ users that should be accurately designed over the optical carrier $\lambda_{n}$, in which we can minimize the objective function $\left\|\mathbf{F}_{\mathrm{opt}}-\mathbf{F}_{\mathrm{RoF}} \mathbf{f}_{\mathrm{OAWG}} \mathbf{F}_{\mathrm{BB}}\right\|_{F}^{2}$ and maximize the beam-steering function $\left|\alpha_{t}^{H}\left(\emptyset_{k, l}\right) \mathbf{F}_{\mathrm{RoF}}^{(n)}\right|^{2}$ at the line of sight propagation path (where the beam-steering angle is located for user $k$ ); here $\sigma$ is defined as an optimization factor that should be chosen to minimize the target function. The transmitter has an optimal digital precoding scheme when the number of RF chains (DACs) is equivalent to the number of antenna elements, where the analog photonic beamformer $\mathrm{F}_{\mathrm{RoF}}$ is omitted from the system structure design, i.e., $N_{r}=$ $M$, which in turn leads to a high-cost hardware implementation and power consumption. Since the mm-wave signals are inherently sparse signals (which is due to the impact of propagation path loss, where few multi-path components $(L)$ are existed at the receiver), conventional RF systems usually use full (optimal) digital precoders to achieve a large coding gain and obtain a high SNR at the receiver, which makes the use of optimal digital precoders impractical at mmwave because of the critical constraints to the design of mmwave transceivers [2]. In practice; in order to minimize interuser interference in ultra-dense small cells and maximize the mutual information between the transmitter and receiver, the ${ }_{H}$ photonic beamformer $\mathbf{F}_{\text {RoF }}$ design for user $k$ should be under the power constraint of $\left\|\mathbf{F}_{\mathrm{RoF}} \mathbf{f}_{\mathrm{OAWG}}\right\|_{F}^{2}=N_{r}$. Now using the identity of $\|A-B\| \geq\|A\|-\|B\| \|$, the objective function $\left\|\mathbf{F}_{\text {opt }}-\mathbf{F}_{\mathrm{RoF}} \mathbf{f}_{\mathrm{OAWG}} \mathbf{F}_{\mathrm{BB}}\right\|_{F}^{2}$ can be further simplified as

$$
\begin{aligned}
\left\|\mathbf{F}_{\mathrm{opt}}-\mathbf{F}_{\mathrm{RoF}} \mathbf{f}_{\mathrm{OAWG}} \mathbf{F}_{\mathrm{BB}}\right\|_{F}^{2} & \geq\|\| \mathbf{F}_{\mathrm{opt}}\|-\| \mathbf{F}_{\mathrm{RoF}} \mathbf{f}_{\mathrm{OAWG}} \mathbf{F}_{\mathrm{BB}} \|^{2} \\
& \geq\left|\sqrt{N_{r} K}-\sqrt{\sigma K}\right|^{2}
\end{aligned}
$$


Thus, when $\sigma=N_{r}$, the objective function has the minimum value, given by $\left\|\mathbf{F}_{\text {opt }}\right\|^{2}-\sigma$, resulting in achievable spectral efficiency expressed as

$R \leq \sum_{k} \sum_{l} \log _{2}\left(1+\frac{\mathrm{snr}}{L K^{2}} M N_{r}(K-1)\left|\alpha_{k, l}\right|^{2}\right)$ bits $/ \mathrm{s} / \mathrm{Hz}$

Notice here that the spatial multiplexing gain (degrees of freedom) extracted from the channel $\mathbf{H}$, is $L K$. In addition, the worst-case for the photonic hybrid precoding is when $N_{r}=K$ and the best-case is when $N_{r}=M$ (like in the case of the optimal digital precoding). Since the photonic beamforming vector improves the signal power of users, there is no need to implement more RF chains at the transceivers.

Special cases: A user at the edge of a cell may experience a low SNR and interference. Therefore, using the approximation $\log _{2}(1+x) \approx x \log _{2}$ e for $x$ small, we obtain

$$
R_{\mathrm{snr} \rightarrow 0}^{(k)} \leq \frac{M N_{r}}{K^{2}}(K-1) \mathrm{snr} \cdot \log _{2} \mathrm{e} \quad \text { bits } / \mathrm{s} / \mathrm{Hz}
$$

At low SNR values, each user can yield a power gain equal to $M N_{r}(K-1) / K^{2}$. Note that when the number of scatters $L$ increases, the power gain does not increase. On the other hand, when increasing the number of transmit antennas and/or the number of optical carriers, the power gain is amplified by the photonic beamformer $\mathbf{F}_{\mathrm{RoF}}$, resulting in the spectral efficiency enhancement. Therefore, sending the users' data streams over multi-carrier frequencies makes the RoF systems maximize the received SNR even if the transmitter has limited channel knowledge. This feature, of course, allows the photonic hybrid precoders to overcome some common problems associated with inter-symbol interference and interuser interference for conventional multi-user MIMO systems at mm-wave [24]. While this problem was practically solved for wideband single-user systems through orthogonal frequency division multiplexing (OFDM), there are still some challenges of implementing the multi-user precoding at mm-wave [25]. With a short wavelength of a mm-wave, it is possible to pack a large number of antennas into the photonic beamformer to overcome the high path loss. For multi-user MIMO systems, the number of transmit antennas greatly exceeds the number of users (i.e, $M \gg K, M \rightarrow \infty$ ), where the row vectors of the channel $\mathbf{H}$ are asymptotically orthogonal. In this case, we have

$$
\frac{\mathbf{H H}^{H}}{M} \approx \mathbf{I}_{K}
$$

Assuming $\alpha_{k, l}(l=1,2, \ldots, L)$ are independent and identical distributed (i.i.d) $\mathcal{C N}(0,1)$ random variables with LOS path to user $k$, the achievable spectral efficiency (10) is approximated as

$$
R^{(k)} \approx \log _{2}\left(1+\operatorname{snr} . M N_{r}\right) \quad \text { bits } / \mathrm{s} / \mathrm{Hz}
$$

As we notice in (17), massive MIMO systems with multicarrier transmission can greatly improve the power gain up to $M N_{r}$, which treats any low SNR and interference. Massive MIMO systems have also the capability of providing steerable beams to compensate large path-loss of mm-wave. However, increasing antenna elements at a microcell base station can create narrower beams and higher gains but may increase the channel estimation errors and pilot contamination [24]. In conventional multi-cell massive MIMO systems, users in neighboring cells may use non-orthogonal pilots because the number of pilot sequences is smaller than the number of users, which results in inter-cell interference and pilot contamination. Since RoF systems deploy a large-scale small cell network, a small number of orthogonal pilot sequences is required to serve a small number of users per cell. Thus, using massive MIMO systems in small cell networks can completely remove the effect of pilot contamination and maximize the spectral efficiency [26].

\section{B. Average Bit Error Rate}

Assuming each user employs a maximum likelihood (ML) detector, the bit error rate (BER) for binary phase-shift keying (BPSK) modulation is expressed as $P_{b}^{(k)} \geq Q\left(\sqrt{2 \gamma_{k}}\right)$, where the effective SNR at user $k\left(\gamma_{k}\right)$, is given by

$$
\gamma_{k}=\sum_{l} \operatorname{snr} \frac{M N_{r}}{L K^{2}}(K-1)\left|\alpha_{k, l}\right|^{2}
$$

Following the derivation in [27, eq. (3.44)] and assuming the complex gains $\alpha_{k, l}$ are i.i.d $\mathcal{C N}(0,1)$ random variables, the average BER for user $k$ can be simplified in the high SNR regime (i. e., snr $>>1$ )) as

$$
\bar{P}_{b}^{(k)} \leq\left(\operatorname{snr} \frac{M N_{r}}{L K^{2}}(K-1)\right)^{-L}
$$

Notice that the diversity order for the underlying scheme is $d=L$, whereas the coding gain is $r=M N_{r}(K-1) / L K^{2}$. Fig 4 plots both the spectral efficiency and the bit error probability of BPSK modulation for both RoF systems and conventional RF systems (where the optical intensity modulator array $\mathbf{f}_{\mathrm{OAWG}}$ is omitted and the RF beamformer $\mathbf{f}_{\mathrm{RF}} \in$ $C^{M \times 1}$ [1] operates at a single carrier, i.e., $n=1$ for $\left.\lambda_{n}\right)$. The performance is evaluated in single-path mm-wave channels (where we assume the number of scatters is very limited, $L=1$ ). Multiplexed data streams are sent from an RAU with $M=16$ antennas to a set of users $(K=3)$, each having a single antenna. In addition, we assume that a zeroforcing precoder is employed at the central office and the number of RF chains and optical carriers, is equal to the number of users i.e., $K \leq N_{r}$. This is due to the spatial multiplexing gain of the described hybrid precoding system, which is limited by $\min \left(N_{r}, K\right)$ for $N_{r} \leq M$ [1]. While we use a mm-wave channel model (4) to simplify our analysis; in our simulation, we perform the Saleh-Valenzuela model with a single propagation cluster, in which the RAU has a uniform square planar array (USPA) with $\sqrt{M} \times \sqrt{M}$ antenna elements [28]. The azimuth and elevation of AoAs/AoDs are uniformly distributed in $[0,2 \pi]$ and $\left[-\frac{\pi}{2}, \frac{\pi}{2}\right]$, respectively.

In this setting, Monte-Carlo simulation is implemented at $10^{5}$ channel realizations, where both spectral efficiency, $R=\log _{2}\left(1+\mathrm{SINR}_{k}\right)$, and BER for the received signal per 
user (1), are analyzed. The results indicate that the photonic hybrid precoders can achieve better interference mitigation in multiuser transmission scenarios compared with conventional RF hybrid precoders [1]. The results show that the RoF systems bring an extra gain over conventional RF systems; which is expected since the photonic beamformer offers a higher power gain than the RF beamformer. This can be deduced directly from (15) and (19), the power and coding gain ratio of the RoF systems to the RF systems is $N_{r}$. Thus, the photonic hybrid precoders can achieve better interference mitigation in multi-user MIMO systems compared with conventional RF hybrid precoders. These findings indicate that: 1) the RoF systems can provide higher data streams per user, resulting in higher data transmission, 2) they can achieve a significant power gain for low SNR and interference scenarios without performing power allocation techniques to maximize the link spectral efficiency, and 3) multi-carrier transmission implies that; multiple broadband signals can be transmitted to multiple users, in such a way that the system dynamically maximizes the coding gain and minimizes the number of RF chains.

\section{CONCLUSION}

In this paper, we proposed a new hybrid precoding structure for RoF systems. The system architecture considers both the flexibility and the robustness to conventional RF precoders inaccuracy. The photonic hybrid precoding systems aim to overcome the large propagation loss and reduce channel estimation cost at mm-wave. Our proposed model addressed the problem of low SNR and interference in small cell scenarios. The simulation results clearly demonstrated that RoF systems can offer a higher coding gain than conventional RF systems when the numbers of RF chains and users are equal. The photonic hybrid precoders can efficiently overcome multi-user precoding design problems at mm-wave, by enabling analog photonic beamformers to increase the power gain and mitigate inter-user interference.

\section{REFERENCES}

[1] A. Alkhateeb, G. Leus, and R. Heath, "Limited feedback hybrid precoding for multi-user millimeter wave systems," IEEE Trans. Wireless Commun., vol. 14, no. 11, pp. 6481-6494, Nov. 2015.

[2] Z. Gao, L. Dai, S. Han, I. Chih-Lin, Z. Wang, and L. Hanzo, "Compressive sensing techniques for next-generation wireless communications," IEEE Wireless Commun., vol. 25, no. 3, pp. 144-153, Jun. 2018.

[3] M. A. L. Sarker, M. F. Kader and D. S. Han, "Rate-Loss Mitigation for a Millimeter-Wave Beamspace MIMO Lens Antenna Array System Using a Hybrid Beam Selection Scheme,” IEEE Sys. J.,pp. 1-4, Jan. 2020.

[4] C. Liu, J. Wang, L. Cheng, M. Zhu, and G.-K. Chang, "Key microwavephotonics technologies for next-generation cloud-based radio access networks," J. Lightw. Technol., vol. 32, no. 20, pp. 3452-3460, Oct. 2014.

[5] H. Fei. Opportunities in 5G networks: A research and development perspective. CRC Press, 2016.

[6] I. Djordjevic, B. Vasic, and M. Neifeld, "LDPC-coded OFDM for optical communication systems with direct detection," IEEE J. Sel. Topics Quantum Electron., vol. 13, no. 5, pp. 1446-1454, Oct. 2007.

[7] L. Beygi, E. Agrell, J. M. Kahn, and M. Karlsson, "Coded modulation for fiber-optic networks: Toward better tradeoff between signal processing complexity and optical transparent reach," IEEE Signal Process. Mag., vol. 31, no. 2, pp. 93-103, Mar. 2014.

[8] M. Žhu, L. Zhang, J. Wang, L. Cheng, C. Liu, and G.-K. Chang, "Radio-over-fiber access architecture for integrated broadband wireless services,"J. Lightw. Technol., vol. 31, no. 23, pp. 3614-3620, Dec. 2013.
[9] T. Komljenovic et al., "Heterogeneous silicon photonic integrated circuits," J. Lightw. Technol., vol. 34, no. 1, pp. 20-35, Jan. 2016.

[10] K. Minoguchi, Y. Nishikawa, M. Oishi, S. Akiba, J. Hirokawa, and M. Ando, "Beam steering scheme of photonic array-antennas for $60 \mathrm{GHz}$ RF signals generated by optical two-tone technique," in Optical Fiber Tech., 2014 OptoElectronics and Commun. Conf. and Australian Conf., 2014, pp. 861-863.

[11] S.B. Guan, S. Djordjevic, N. K. Fontaine, L. Zhou, S. Ibrahim, R. P. Scott, D. Geisler, Z. Ding, and S. J. B. Yoo, "CMOS Compatible Reconfigurable Silicon Photonic Lattice Filters using Cascaded Unit Cells for RF-Photonic Processing," IEEE J. Sel. Topics Quantum Electron., vol. PP, pp. 1-1, Aug. 2014.

[12] S. S. Djordjevic, L. W. Luo, S. Ibrahim, N. K. Fontaine, C. B. Poitras, B. B. Guan, L. J. Zhou, K. Okamoto, Z. Ding, M. Lipson, and S. J. B. Yoo, "Fully Reconfigurable Silicon Photonic Lattice Filters With Four Cascaded Unit Cells," IEEE Photonics Tech. Lett., vol. 23, pp. 42-44, Jan. 2011

[13] A. Beling, A. S. Cross, M. Piels, J. Peters, Y. Fu, Q. Zhou, J. E. Bowers, and J. C. Campbell, "High-power highspeed waveguide photodiodes and photodiode arrays heterogeneously integrated on Silicon-on-insulator," Proc. IEEE Optical Fiber Commun. Conf. and Exposition and the National Fiber Optic Engineers Conf., pp. 1-3, Mar. 2013.

[14] E. Rouvalis, C. C. Renaud, D. G. Moodie, M. J. Robertson, and A. J. Seeds, "Traveling-wave uni-traveling carrier photodiodes for continuous wave Thz generation," Opt. Exp., vol. 18, no. 11, pp. 11105-11110, May. 2010.

[15] H. Ito, S. Kodama, Y. Muramoto, T. Furuta, T. Nagatsuma, and T. Ishibashi, "High-speed and high-output InP-InGaAs unitraveling-carrier photodiodes," IEEE J. Sel. Topics Quantum Electron., vol. 10, pp. 709727, Oct. 2004.

[16] A.A.M. Saleh, and R.A. Valenzuela, "A Statistical Model for Indoor Multipath Propagation," IEEE J. on Sel. Areas in Commun., vol.5, no.2, pp.128-137, Feb. 1987.

[17] H. Q. Ngo, “Massive MIMO: Fundamentals and system designs”, Ph.D. dissertation, Linkoping University, 2015.

[18] D. Tse and P. Viswanath, Fundamentals of Wireless Communications., Cambridge, U.K.: Cambridge Univ. Press, 2005.

[19] V. Raghavan and A. Sayeed, "Sublinear capacity scaling laws for sparse MIMO channels," IEEE Trans. Inf. Theory, vol. 57, no. 1, pp. 345-364, Jan. 2011

[20] M. Attygalle and D. Stepanov, "Photonic Technique for Phase Control of Microwave to Terahertz Signals," IEEE Trans. Microw. Theory Techn, vol. 62, no. 6, pp. 1381-1386, Jun. 2014.

[21] C. Vagionas et al., "A 6-Band 12Gb/s IFoF/V-Band Fiber-Wireless Fronthaul Link Using an InP Externally Modulated Laser," Proc. IEEE European Conf. on Optical Commun. (ECOC), pp. 1-3, May. 2018.

[22] Y. Zhu, Y. Wu, H. Xu, C. Browning, L. P. Barry and Y. Yu, "Experimental Demonstration of a WDM-RoF Based Mobile Fronthaul With fOFDM Signals by Using Directly Modulated 3s-DBR Laser,' J. Lightw. Technol., vol. 37, no. 16, pp. 3875-3881, Aug. 2019.

[23] ME. Belkin, et al.,'Design and Optimization of Photonics-Based Beamforming Networks for Ultra-Wide mmWave-Band Antenna Arrays", Wireless Communication Networks, Array Pattern Optimization, Nov. 2018.

[24] L. Lu, G. Y. Li, A. L. Swindlehurst, A. Ashikhmin, and R. Zhang, "An overview of massive MIMO: benefits and challenges," IEEE J. Sel. Topics Signal Process, vol. 8, pp. 742- 758, Oct. 2014.

[25] X. Yu, J. Zhang, and K. B. Letaief, "Alternating minimization for hybrid precoding in multi-user OFDM mmWave Systems," Proc. IEEE Asilomar Conf. on Signals, Systems, and Computers, pp. 281-285, Nov. 2016.

[26] A. Kazerouni, F. J. Lopez-Martinez, and A. Goldsmith, "Increasing capacity in massive MIMO cellular networks via small cells", 2014 IEEE Globecom Workshops (GC Wkshps), Austin, TX, Dec. 2014, pp. 358-363.

[27] A. Paulraj, R. Nabar, and D. Gore, Introduction to Space-Time Wireless Communications. Cambridge, U.K.: Cambridge Univ. Press, 2003.

[28] T. S. Rappaport, R. W. Heath, R. C. Daniels, and J. N. Murdock, Millimeter Wave Wireless Communications. New York, USA: Pearson Education, 2014. 